MATHEMATICS OF COMPUTATION

Volume 76, Number 258, April 2007, Pages 1039-1057

S 0025-5718(06)01914-4

Article electronically published on December 4, 2006

\title{
QUATERNIONIC MANIN SYMBOLS, BRANDT MATRICES, AND HILBERT MODULAR FORMS
}

\author{
LASSINA DEMBÉLÉ
}

\begin{abstract}
In this paper, we propose a generalization of the algorithm we developed previously. Along the way, we also develop a theory of quaternionic $M$ symbols whose definition bears some resemblance to the classical $M$-symbols, except for their combinatorial nature. The theory gives a more efficient way to compute Hilbert modular forms over totally real number fields, especially quadratic fields, and we have illustrated it with several examples. Namely, we have computed all the newforms of prime levels of norm less than 100 over the quadratic fields $\mathbb{Q}(\sqrt{29})$ and $\mathbb{Q}(\sqrt{37})$, and whose Fourier coefficients are rational or are defined over a quadratic field.
\end{abstract}

\section{INTRODUCTION}

This paper is a generalization of 4 (see also the author's thesis [3]). It presents a new approach to the theory of Brandt matrices in order to compute Hilbert modular forms. To give a brief discussion of this approach, let $F$ be a totally real number field of even degree and narrow class number one-we will explain later how those conditions can be relaxed - and let B be the unique (up to isomorphism) totally definite quaternion algebra whose ramification is only at infinity. We let $\mathfrak{N}$ be an integral ideal in $F$. By the Jacquet-Langlands correspondence, we know that any Hilbert modular form of level $\mathfrak{N}$ and arbitrary weight $\underline{k}$ comes from an automorphic form of the same level and weight on B (see Section 3 and reference therein). The algorithm in Pizer [12, which has been the most used so far when it comes to computing modular forms on fields larger than $\mathbb{Q}$ is based on the knowledge of the invariants of an Eichler order of level $\mathfrak{N}$ in $\mathrm{B}$, such as its class number, representatives of its ideal classes, and the left or right orders of those ideals; see, for example, Consani and Scholten [1, Pizer [12] and Socrates and Whitehouse [15]. The main disadvantage of this approach is that one needs to throw away almost everything and start all over again when the level changes. Our approach, instead, is based on using invariants of the quaternion algebra B itself. So, the knowledge of the Eichler order becomes rather virtual. From a computational point of view, this has tremendous advantages as one can store a huge amount of data from the start, and the computations which are required for each level amount to finding some local embeddings of a set of representatives of the types of B.

Though, the aim of this paper was primarily computational, some of the issues we address certainly have some theoretical interest. Indeed, our approach to the

Received by the editor April 8, 2004 and, in revised form, January 18, 2006.

2000 Mathematics Subject Classification. Primary 11-xx; Secondary 11Gxx.

Key words and phrases. Hilbert modular forms, automorphic forms, Brandt matrices.

(C)2006 American Mathematical Society

Reverts to public domain 28 years from publication 
Brandt matrices suggests that one can give a purely algebraic description of the Hecke module $S_{\underline{k}}^{\mathrm{B}}(\mathfrak{N})$, the space of automorphic forms of level $\mathfrak{N}$ and weight $\underline{k}$ on B. This leads us to the notion of quaternionic Manin symbols. The definition of the Hecke module of the quaternionic Manin symbols has a lot of resemblance to the one of the usual Manin symbols when $F=\mathbb{Q}$ (see Merel 11] or Stein [16]). Unfortunately, the only way one can relate this module to the holomorphic part of the cuspidal cohomology of the Hilbert modular variety $X_{0}(\mathfrak{N})$ seems to be by means of the Jacquet-Langlands correspondence. The similarities of the quaternionic $M$-symbols with the usual $M$-symbols over $\mathbb{Q}$ suggests that one could implement them in a package similar to the Magma package of Stein [16, 9] for a wide range of number fields, especially real quadratic fields. One of the applications we have in mind for such an algorithm would be to gather more numerical evidence about the Serre conjecture for totally real number fields.

As can be seen easily, the definition of the automorphic forms in this paper is a special case of the theory of algebraic modular forms in Gross 7 . So, we think that quaternionic $M$-symbols could also help in approaching those forms, at least from a computational standpoint.

The paper is organized as follows. Sections 1 and 2 recall preliminaries on Hilbert modular forms and automorphic forms on definite quaternion algebras, together with the Jacquet-Langlands correspondence which allows one to go from one side to the other. In Section 3, we describe the Brandt matrices, and in Section 4 we show how one can add a character to that definition. In Section 5, we define quaternionic $M$-symbols and give their basic properties. The final two sections give numerical examples on the theory we have developed; namely, we give tables of all the eigenforms of parallel weight $(2,2)$ and prime level $\mathfrak{N}$ of norm less than 100 , whose coefficients are rationals or defined over a quadratic field, for the fields $F=\mathbb{Q}(\sqrt{29})$ and $F=\mathbb{Q}(\sqrt{37})$.

\section{HilbERT MODUlar FORMS}

We fix a totally real number field $F$ of even degree $g$. We assume that the narrow class number of $F$ is one. We let $I$ be the set of all real embeddings of $F$. For each $\tau \in I$, we denote the corresponding embedding into $\mathbb{R}$ by $a \mapsto a^{\tau}$. Also, we let $\mathcal{O}_{F}$ be the ring of integers of $F$, and $\delta$ its different. For an integral ideal $\mathfrak{p}$ of $F$, we denote by $F_{\mathfrak{p}}$ and $\mathcal{O}_{F, \mathfrak{p}}$ the completions of $F$ and $\mathcal{O}_{F}$, respectively, at $\mathfrak{p}$. We say that an element $a \in F$ is totally positive if, for all $\tau \in I, a^{\tau}>0$. We then denote this by $a \gg 0$ and, for every subring $A$ of $F$, we let $\mathbf{G L}_{2}^{+}(A)$ be all the invertible 2 by 2 matrices with coefficients in $A$, with totally positive determinant. We fix an integral ideal $\mathfrak{N}$ of $F$, and put

$$
\Gamma_{0}(\mathfrak{N})=\left\{\gamma=\left(\begin{array}{ll}
a & b \\
c & d
\end{array}\right) \in \mathbf{G L}_{2}^{+}\left(\mathcal{O}_{F}\right): c \in \mathfrak{N}\right\} .
$$

Let $\underline{k}=\left(k_{\tau}\right)_{\tau \in I} \in \mathbb{Z}^{I}$ be an integer vector whose components have the same parity, $k_{\tau} \geq 2$. Let $\mathcal{H}$ denote the Poincaré upper half-plane. We recall that $\Gamma_{0}(\mathfrak{N})$ acts on the set of functions $f: \mathcal{H}^{I} \rightarrow \mathbb{C}$ by

$$
f \|_{\underline{k}} \gamma(z)=\left(\prod_{\tau \in I} \operatorname{det} \gamma_{\tau}^{k_{\tau} / 2}\left(c^{\tau} z_{\tau}+d^{\tau}\right)^{-k_{\tau}}\right) f\left(\left(\frac{a^{\tau} z_{\tau}+b^{\tau}}{c^{\tau} z_{\tau}+d^{\tau}}\right)_{\tau \in I}\right) .
$$


Definition 1. A Hilbert modular form of level $\mathfrak{N}$ and weight $\underline{k}$ is a holomorphic function $f: \mathcal{H}^{I} \rightarrow \mathbb{C}$ such that

$$
f \|_{\underline{k}} \gamma=f \quad \text { for all } \gamma \in \Gamma_{0}(\mathfrak{N}) .
$$

Let $f$ be a Hilbert modular form. Then

$$
f(z+\mu)=f(z) \quad \text { for all } z \in \mathcal{H}^{I}, \mu \in \mathcal{O}_{F} .
$$

Therefore, $f$ admits a Fourier expansion which, by the Koecher principle, is of the form

$$
f(z)=\sum_{\nu \in \delta^{-1}} a_{\nu} e^{2 \pi i \operatorname{Tr}(\nu z)}
$$

with $\nu=0$ or $\nu \gg 0$. Also, since $f$ is invariant under the action of matrices of the form $\left(\begin{array}{cc}\varepsilon & 0 \\ 0 & \varepsilon^{-1}\end{array}\right)$, with $\varepsilon \in \mathcal{O}_{F}^{\times}$, we see that $a_{\nu}=\mathbf{N}(\varepsilon)^{\underline{k} / 2} a_{\varepsilon^{2} \nu}$. For any integral ideal $\mathfrak{a}$ of $F$, we choose $\nu \gg 0$ such that $\mathfrak{a}=(\nu) \delta$ and put

$$
c(\mathfrak{a}, f)=a_{\nu} .
$$

This is a well-defined coefficient. For all $\gamma \in \mathbf{G L}_{2}^{+}(F)$, let $a_{0}^{\gamma}$ be the first coefficient in the Fourier expansion of $f \|_{\underline{k}} \gamma$. We say that $f$ is a cusp form if $a_{0}^{\gamma}=0$, for all $\gamma \in \mathbf{G L}_{2}^{+}(F)$. We denote the space of all cusp forms by $S_{\underline{k}}(\mathfrak{N})$.

The Petersson inner product on the space of cusp forms is given by

$$
\langle f, g\rangle:=\frac{1}{\mu\left(\Gamma_{0}(\mathfrak{N}) \backslash \mathcal{H}^{I}\right)} \int_{\Gamma_{0}(\mathfrak{N}) \backslash \mathcal{H}^{I}} f(z) \overline{g(z)} y^{\underline{k}} d \mu(z),
$$

where

$$
d \mu(z)=\prod_{\tau \in I} \frac{d y_{\tau}}{y_{\tau}^{2}}, \quad \text { and } y^{\underline{k}}=\prod_{\tau \in I} y_{\tau}^{k_{\tau}}, \quad \text { for } z_{\tau}=x_{\tau}+i y_{\tau} .
$$

Hecke operators. Let $\mathfrak{p}$ be a prime ideal of $F$ such that $(\mathfrak{p}, \mathfrak{N})=1$, and $\pi_{\mathfrak{p}}$ a uniformizer of $\mathfrak{p}$. We write the disjoint union

$$
\Gamma_{0}(\mathfrak{N})\left(\begin{array}{cc}
1 & 0 \\
0 & \pi_{\mathfrak{p}}
\end{array}\right) \Gamma_{0}(\mathfrak{N})=\coprod \Gamma_{0}(\mathfrak{N}) \alpha_{i},
$$

and define the action of the Hecke operators $T_{\mathfrak{p}}$ on $S_{\underline{k}}(\mathfrak{N})$ by

$$
f\left\|T_{\mathfrak{p}}=\sum f\right\|_{\underline{k}} \alpha_{i}
$$

The operators $T_{\mathfrak{p}}$ generate a commutative (finite) $\mathbb{Z}$-subalgebra of $\operatorname{End}\left(S_{\underline{k}}(\mathfrak{N})\right)$. We denote it by $\mathbf{T}_{\underline{k}}(\mathfrak{N})$ and call it the Hecke algebra of level $\mathfrak{N}$.

Definition 2. A Hecke eigenform $f$ is an eigenvector of $\mathbf{T}_{\underline{k}}(\mathfrak{N})$. We say that $f$ is normalized if $c\left(\mathcal{O}_{F}, f\right)=1$.

It is a result of Shimura that, when $f$ is a normalized eigenform, its eigenvalues are given by the $c(\mathfrak{a}, f)$, for $\mathfrak{a}$ running over the integral ideals in $F$, and that they generate a number field ([14).

We denote by $S_{\underline{k}}^{\text {old }}(\mathfrak{N})$ the subspace of $S_{\underline{k}}(\mathfrak{N})$ generated by all cusp forms of the form $f(d z)$, where $d \in \mathfrak{M}$ and $f \in S_{\underline{k}}\left(\mathfrak{N M}^{-1}\right)$, for $\mathfrak{M}$ a nontrivial divisor of $\mathfrak{N}$. We denote the orthogonal complement of $S_{\underline{k}}^{\text {old }}(\mathfrak{N})$, with respect to the Petersson inner product, by $S_{\underline{k}}^{\text {new }}(\mathfrak{N})$, and call it the subspace of newforms. We also let $\mathbf{T}_{\underline{k}}^{\text {new }}(\mathfrak{N})$ be the part of Hecke algebra which acts on the space of newforms. 


\section{Automorphic Forms on Definite quaternion Algebras AND THE JACQUET-LANGLANDS CORRESPONDENCE}

In this section, we let B be the unique (up to isomorphism) totally definite quaternion algebra of center $F$ that ramifies only at infinite places (this is possible because $F$ has even degree). We fix a maximal order $\mathrm{R}$ in $\mathrm{B}$, and let $\mathrm{R}_{\mathfrak{N}}$ be an Eichler order of level $\mathfrak{N}$ contained in $\mathrm{R}$. We fix a Galois extension $K$ of $F$ contained in $\mathbb{C}$, which splits $\mathrm{B}$. We also fix an isomorphism $\mathrm{B} \otimes_{F} K \cong \mathrm{M}_{2}(K)^{I}$, and let $j: \mathrm{B}^{\times} \hookrightarrow \mathbf{G L}_{2}(\mathbb{C})^{I}$ be the resulting embedding.

We fix a vector $\underline{k} \in \mathbb{Z}^{I}$ such that $k_{\tau} \geq 2$ for all $\tau$, with all the components having the same parity. Set $\underline{t}=(1, \ldots, 1)$ and $\underline{m}=\underline{k}-2 \underline{t}$, then choose $\underline{v} \in \mathbb{Z}^{I}$ such that each $v_{\tau} \geq 0, v_{\tau}=0$ for some $\tau$, and $\underline{m}+2 \underline{v}=n \underline{t}$ for some nonnegative $n \in \mathbb{Z}$. For every nonnegative integer $a, b \in \mathbb{Z}$, we let $\mathbf{S}_{a, b}(\mathbb{C})$ denote the right $\mathrm{M}_{2}(\mathbb{C})$-module $\operatorname{Sym}^{a}\left(\mathbb{C}^{2}\right)$ (the $a$ th symmetric power of the standard right $\mathrm{M}_{2}(\mathbb{C})$-module $\mathbb{C}^{2}$ ) with the $\mathrm{M}_{2}(\mathbb{C})$ action:

$$
x \cdot m:=(\operatorname{det} m)^{b} x \operatorname{Sym}^{a}(m) .
$$

Then, we define

$$
L_{\underline{k}}=\bigotimes_{\tau \in I} \mathbf{S}_{m_{\tau}, v_{\tau}}(\mathbb{C}) .
$$

We let $G=\operatorname{Res}_{F / \mathbb{Q}}\left(\mathrm{B}^{\times}\right)$be the algebraic group obtained by restriction of scalars. Via the obvious extension of $j, G(\mathbb{R})$ acts on $L_{\underline{k}}$. On the complex space of functions $f: G(\mathbb{Q}) \backslash G(\mathbb{A}) \rightarrow L_{\underline{k}}$, we define an action of $G(\mathbb{A})$ by

$$
\left(f \|_{\underline{k}} u\right)(g):=f(g u) u_{\infty}^{-1}, \quad g, u \in G(\mathbb{A}) .
$$

Similarly, on the space of functions $f: G\left(\mathbb{A}_{f}\right) / \hat{\mathrm{R}}_{\mathfrak{N}}^{\times} \rightarrow L_{\underline{k}}$, we define an action of $G(\mathbb{Q})$ by

$$
\left(f \|_{\underline{k}} \gamma\right)(g):=f(\gamma g) \gamma, \quad g \in G(\mathbb{A}), \gamma \in G(\mathbb{Q}) .
$$

Definition 3. The space of automorphic forms of level $\mathfrak{N}$ and weight $\underline{k}$ on $\mathrm{B}$ is

$$
S_{\underline{k}}^{\mathrm{B}}(\mathfrak{N}):=\left\{f: G(\mathbb{Q}) \backslash G(\mathbb{A}) \rightarrow L_{\underline{k}}: f \|_{\underline{k}} u=f, u \in G(\mathbb{R}) \times \hat{\mathrm{R}}_{\mathfrak{N}}^{\times}\right\} .
$$

Equivalently, we can define the space of automorphic forms as

$$
S_{\underline{k}}^{\mathrm{B}}(\mathfrak{N})=\left\{f: G\left(\mathbb{A}_{f}\right) / \hat{\mathrm{R}}_{\mathfrak{N}}^{\times} \rightarrow L_{\underline{k}}: f \|_{\underline{k}} \gamma=f, \quad \gamma \in G(\mathbb{Q})\right\} .
$$

Hecke operators. Take $u \in G\left(\mathbb{A}_{f}\right)$ and write the finite disjoint union

$$
\hat{\mathrm{R}}_{\mathfrak{N}}^{\times} u \hat{\mathrm{R}}_{\mathfrak{N}}^{\times}=\coprod \hat{\mathrm{R}}_{\mathfrak{N}}^{\times} u_{i}
$$

We define the Hecke operator $\left[\hat{\mathrm{R}}_{\mathfrak{N}}^{\times} u \hat{\mathrm{R}}_{\mathfrak{N}}^{\times}\right]$by

$$
\begin{aligned}
{\left[\hat{\mathrm{R}}_{\mathfrak{N}}^{\times} u \hat{\mathrm{R}}_{\mathfrak{N}}^{\times}\right]: S_{\underline{k}}^{\mathrm{B}}(\mathfrak{N}) } & \rightarrow S_{\underline{k}}^{\mathrm{B}}(\mathfrak{N}), \\
f & \mapsto \sum_{i} f \|_{\underline{k}} u_{i} .
\end{aligned}
$$

Let $\mathfrak{p}$ be a prime ideal of $F$ such that $(\mathfrak{p}, \mathfrak{N})=1$, and $\pi_{\mathfrak{p}}$ a uniformizer of $\mathfrak{p}$. We let

$$
T_{\mathfrak{p}}:=\left[\hat{\mathrm{R}}_{\mathfrak{N}}^{\times}\left(\begin{array}{cc}
1 & 0 \\
0 & \pi_{\mathfrak{p}}
\end{array}\right) \hat{\mathrm{R}}_{\mathfrak{N}}^{\times}\right]
$$

The operators $T_{\mathfrak{p}}$ generate a commutative (finite) $\mathbb{Z}$-subalgebra of $\operatorname{End}\left(S_{\underline{k}}^{\mathrm{B}}(\mathfrak{N})\right.$ ), we denote it by $\mathbf{T}_{\underline{k}}^{\mathrm{B}}(\mathfrak{N})$ and call it the Hecke algebra of level $\mathfrak{N}$. 
Let $\mu_{\mathrm{B}}: \mathrm{B} \rightarrow \mathbb{Q}_{+}$be the composition of the norm map on B with the norm map on $F$. We denote by $\mu_{\mathrm{B}, \mathbb{A}}: \mathrm{B}_{\mathbb{A}} \rightarrow \mathbb{R}_{+}$its natural extension to $\mathrm{B}_{\mathbb{A}}$. As in Taylor [17, we can find a bilinear pairing

$$
\langle,\rangle: L_{\underline{k}} \rightarrow \mathbb{C}
$$

such that

$$
\langle x \alpha, y \alpha\rangle=\mu_{\mathrm{B}}(\alpha)^{n}\langle x, y\rangle, \alpha \in \mathrm{B}^{\times} .
$$

We then define a bilinear pairing on $S_{\underline{k}}^{\mathrm{B}}(\mathfrak{N})$ by

$$
\langle f, g\rangle:=\sum_{x \in X_{0}^{\mathrm{B}}(\mathfrak{N})} \mu_{\mathrm{B}, \mathbb{A}}(x)^{n}\langle f(x), g(x)\rangle,
$$

where

$$
X_{0}^{\mathrm{B}}(\mathfrak{N})=G(\mathbb{Q}) \backslash G\left(\mathbb{A}_{f}\right) / \hat{\mathrm{R}}_{\mathfrak{N}}^{\times} .
$$

This bilinear pairing is not Hecke equivariant, but one can show that

$$
\left\langle f \|_{\underline{k}}\left[\hat{\mathrm{R}}_{\mathfrak{N}}^{\times} u \hat{\mathrm{R}}_{\mathfrak{N}}^{\times}\right], g\right\rangle=\mu_{\mathrm{B}, \mathbb{A}}(u)^{n}\left\langle f, g \|_{\underline{k}}\left[\hat{\mathrm{R}}_{\mathfrak{N}}^{\times} u^{-1} \hat{\mathrm{R}}_{\mathfrak{N}}^{\times}\right]\right\rangle .
$$

See Taylor [17] for more details, see also Gross [7, Proposition 1.4] on how to obtain such a symmetric positive definite bilinear pairing in general. Analogously to the previous section, we define the spaces $S_{\underline{k}}^{\mathrm{B}, \text { old }}(\mathfrak{N})$ and $S_{\underline{k}}^{\mathrm{B}, \text { new }}(\mathfrak{N})$, and the Hecke algebra $\mathbf{T}_{\underline{k}}^{\mathrm{B}, \text { new }}(\mathfrak{N})$ using this bilinear pairing. By [7, Proposition 1.4], the pairing is well defined up to scaling by a factor in $\mathbb{Q}_{+}^{\times}$. So the space of newforms does not depend on the choice of this pairing.

Since the ramification of $\mathrm{B}$ is only at infinite places, the Jacquet-Langlands correspondence translates as follows.

Theorem 1 (Jacquet-Langlands). There is an isomorphism of $\mathbb{Z}$-algebras

$$
\mathbf{T}_{\underline{k}}^{\mathrm{B}, \text { new }}(\mathfrak{N}) \cong \mathbf{T}_{\underline{k}}^{\text {new }}(\mathfrak{N}),
$$

and a compatible isomorphism

$$
S_{\underline{k}}^{\mathrm{B}, \text { new }}(\mathfrak{N}) \cong S_{\underline{k}}^{\text {new }}(\mathfrak{N})
$$

of Hecke modules.

Proof. See Jacquet-Langlands [8, Section 16] and Gelbart [6, Chap. 9].

Theorem 1 asserts that one obtains all the newforms on $\mathbf{G L}_{2} / F$ by computing the ones that live on $\mathrm{B}^{\times} / F$. Our next goal is to provide an efficient algorithm which computes the latter space.

\section{BRANDT MATRICES}

We will now bring the Hecke module $S_{k}^{\mathrm{B}}(\mathfrak{N})$ in a form that lends itself better to computation. We keep the notation of the previous section. Let $h$ be the class number of $\mathrm{B}$, and let $\left(g_{\alpha}\right)_{1 \leq \alpha \leq h}$ be a set of finite idèles that generate the right ideal classes of R.

For each $\alpha$, we let $I_{\alpha}$ be the right R-ideal generated by $g_{\alpha}$ and $\mathrm{R}_{\alpha}$ its left (maximal) order. We assume, without loss of generality, that $\mathrm{R}_{1}=\mathrm{R}$, and fix local isomorphisms $\mathrm{R}_{\mathfrak{p}} \cong \mathrm{M}_{2}\left(\mathcal{O}_{F, \mathfrak{p}}\right)$. Via the resulting isomorphism $\hat{\mathrm{R}}_{\alpha}^{\times} \cong \mathbf{G L}_{2}\left(\hat{\mathcal{O}}_{F}\right)$, $\hat{\mathrm{R}}_{\alpha}^{\times}$acts transitively on $\mathbf{P}^{1}\left(\mathcal{O}_{F} / \mathfrak{N}\right)$. We put $\hat{\mathrm{R}}_{\mathfrak{N}, \alpha}=g_{\alpha} \hat{\mathrm{R}}_{\mathfrak{N}} g_{\alpha}^{-1}$, and let $\infty_{\alpha}$ be the point whose stabilizer is $\hat{\mathrm{R}}_{\mathfrak{N}, \alpha}^{\times}$. 
We define the space of automorphic forms of level $\mathfrak{N}$ and weight $\underline{k}$ on the order $\mathrm{R}_{\alpha}$ by

$$
S_{\underline{k}}\left(\mathrm{R}_{\alpha}, \mathfrak{N}\right)=\left\{f: \mathbf{P}^{1}\left(\mathcal{O}_{F} / \mathfrak{N}\right) \rightarrow L_{\underline{k}}: f \|_{\underline{k}} \gamma=f, \quad \gamma \in \mathrm{R}_{\alpha}^{\times}\right\},
$$

with

$$
f \|_{\underline{k}} \gamma(x):=f(\gamma x) \gamma .
$$

As in Definition 3] we can equivalently define the space $S_{\underline{k}}\left(\mathrm{R}_{\alpha}, \mathfrak{N}\right)$ as

$$
S_{\underline{k}}\left(\mathrm{R}_{\alpha}, \mathfrak{N}\right)=\left\{f: \mathrm{R}_{\alpha}^{\times} \backslash G(\mathbb{R}) \times \hat{\mathrm{R}}_{\alpha}^{\times} \rightarrow L_{\underline{k}}: f \|_{\underline{k}} u=f, u \in G(\mathbb{R}) \times \hat{\mathrm{R}}_{\mathfrak{N}, \alpha}^{\times}\right\} .
$$

We will be using both definitions with no distinction.

We now define Hecke operators between such spaces. For each $\alpha, \beta$, let $I_{\alpha, \beta}=$ $I_{\alpha} I_{\beta}^{-1}$. Then, $I_{\alpha, \beta}$ is an ideal in B whose left order (resp. right order) is $\mathrm{R}_{\alpha}$ (resp. $\left.\mathrm{R}_{\beta}\right)$. Now, take $u \in \hat{\mathrm{R}}_{\alpha}\left(g_{\alpha} g_{\beta}^{-1}\right)$ and write the (finite) disjoint union

$$
\hat{\mathrm{R}}_{\mathfrak{N}, \alpha}^{\times} u \hat{\mathrm{R}}_{\mathfrak{N}, \beta}^{\times}=\coprod_{\nu} u_{\nu} \hat{\mathrm{R}}_{\mathfrak{N}, \beta}^{\times},
$$

and for each $x \in G(\mathbb{R}) \times \hat{\mathrm{R}}_{\alpha}^{\times}$, put

$$
\mathbf{N}(x, \alpha, \beta, u)=\left\{u_{\nu}: x u_{\nu}=\gamma_{\nu} x_{\nu} \text { for some } \gamma_{\nu} \in \mathrm{B}^{\times}, x_{\nu} \in G(\mathbb{R}) \times \hat{\mathrm{R}}_{\beta}^{\times}\right\} .
$$

Take $f \in S_{\underline{k}}\left(\mathrm{R}_{\beta}, \mathfrak{N}\right)$ and $x \in G(\mathbb{R}) \times \hat{\mathrm{R}}_{\alpha}^{\times}$, and put

$$
f\left\|_{\underline{k}}\left[\hat{\mathrm{R}}_{\mathfrak{N}, \alpha}^{\times} u \hat{\mathrm{R}}_{\mathfrak{N}, \beta}^{\times}\right](x)=\sum_{\nu \in \mathbf{N}(x, \alpha, \beta, u)} f\right\|_{\underline{k}} u_{\nu}(x),
$$

where we choose $\gamma_{\nu} \in \mathrm{B}^{\times}$and $x_{\nu} \in G(\mathbb{R}) \times \hat{\mathrm{R}}_{\beta}^{\times}$such that $x u_{\nu}=\gamma_{\nu} x_{\nu}$, and put

$$
f \|_{\underline{k}} u_{\nu}(x)=f\left(x_{\nu}\right) .
$$

If $x u_{\nu}=\gamma_{\nu} x_{\nu}=\gamma_{\nu}^{\prime} x_{\nu}^{\prime}$, then $\gamma_{\nu}^{-1} \gamma_{\nu}^{\prime} \in \mathrm{R}_{\beta}^{\times}$. And, since $f$ is left $\mathrm{R}_{\beta}^{\times}$-invariant, we see that $f\left(x_{\nu}\right)=f\left(x_{\nu}^{\prime}\right)$. So, $f \|_{\underline{k}} u_{\nu}(x)$ is well defined. Furthermore, multiplication to the right of the $u_{\nu}$ 's by elements in $\hat{\mathrm{R}}_{\mathfrak{N}, \beta}^{\times}$does not affect their cosets, and multiplication to the left by elements in $\hat{\mathrm{R}}_{\mathfrak{N}, \alpha}^{\times}$will induce a permutation of those cosets. As a result, we get that $f \|_{\underline{k}}\left[\hat{\mathrm{R}}_{\mathfrak{N}, \alpha}^{\times} u \hat{\mathrm{R}}_{\mathfrak{N}, \beta}^{\times}\right]$is well defined and belongs to $S_{\underline{k}}\left(\mathrm{R}_{\alpha}, \mathfrak{N}\right)$. Thus, we have a map

$$
\begin{aligned}
{\left[\hat{\mathrm{R}}_{\mathfrak{N}, \alpha}^{\times} u \hat{\mathrm{R}}_{\mathfrak{N}, \beta}^{\times}\right]: S_{\underline{k}}\left(\mathrm{R}_{\beta}, \mathfrak{N}\right) } & \rightarrow S_{\underline{k}}\left(\mathrm{R}_{\alpha}, \mathfrak{N}\right), \\
f & \mapsto f \|_{\underline{k}}\left[\hat{\mathrm{R}}_{\mathfrak{N}, \alpha}^{\times} u \hat{\mathrm{R}}_{\mathfrak{N}, \beta}^{\times}\right],
\end{aligned}
$$

which we call the Hecke operator $\left[\hat{\mathrm{R}}_{\mathfrak{N}, \alpha}^{\times} u \hat{\mathrm{R}}_{\mathfrak{N}, \beta}^{\times}\right]$. We can now state the following result.

Theorem 2. The map

$$
\begin{aligned}
S_{\underline{k}}^{\mathrm{B}}(\mathfrak{N}) & \rightarrow \bigoplus_{\alpha=1}^{h} S_{\underline{k}}\left(\mathrm{R}_{\alpha}, \mathfrak{N}\right), \\
f & \mapsto\left(f_{\alpha}\right)_{\alpha},
\end{aligned}
$$

where

$$
f_{\alpha}\left(x \cdot \infty_{\alpha}\right)=f\left(x g_{\alpha}\right), \quad x \in \hat{\mathrm{R}}_{\alpha}^{\times},
$$

is an isomorphism of Hecke modules. 
Proof. We first recall that every element in $S_{\underline{k}}^{\mathrm{B}}(\mathfrak{N})$ is completely determined by its values on the finite set

$$
G(\mathbb{Q}) \backslash G\left(\mathbb{A}_{f}\right) / \hat{\mathrm{R}}_{\mathfrak{N}}^{\times}=\mathrm{B}^{\times} \backslash \hat{\mathrm{B}}^{\times} / \hat{\mathrm{R}}_{\mathfrak{N}}^{\times}
$$

Now, since B has class number $h$, we can write the disjoint union

$$
\hat{\mathrm{B}}^{\times}=\coprod_{\alpha=1}^{h} \mathrm{~B}^{\times} g_{\alpha} \hat{\mathrm{R}}^{\times},
$$

hence

$$
\begin{aligned}
\mathrm{B}^{\times} \backslash \hat{\mathrm{B}}^{\times} / \hat{\mathrm{R}}_{\mathfrak{N}}^{\times} & =\mathrm{B}^{\times} \backslash\left(\coprod_{\alpha=1}^{h} \mathrm{~B}^{\times} g_{\alpha} \hat{\mathrm{R}}^{\times}\right) / \hat{\mathrm{R}}_{\mathfrak{\mathfrak { N }}}^{\times} \\
& =\coprod_{\alpha=1}^{h} \mathrm{~B}^{\times} \backslash\left(\mathrm{B}^{\times} g_{\alpha} \hat{\mathrm{R}}^{\times}\right) / \hat{\mathrm{R}}_{\mathfrak{N}}^{\times}
\end{aligned}
$$

Now, it is not hard to verify that the map

$$
\begin{aligned}
\mathrm{B}^{\times} \backslash \mathrm{B}^{\times} g_{\alpha} \hat{\mathrm{R}}^{\times} / \hat{\mathrm{R}}_{\mathfrak{N}}^{\times} & \rightarrow \mathrm{R}_{\alpha}^{\times} \backslash \hat{\mathrm{R}}_{\alpha}^{\times} / \hat{\mathrm{R}}_{\mathfrak{N}, \alpha}^{\times}, \\
\gamma g_{\alpha} x & \mapsto \mathrm{R}_{\alpha}^{\times}\left(g_{\alpha} x g_{\alpha}^{-1}\right) \hat{\mathrm{R}}_{\mathfrak{N}, \alpha}^{\times}
\end{aligned}
$$

is a bijection. By recalling that $\hat{\mathrm{R}}_{\alpha}^{\times}$acts transitively on $\mathbf{P}^{1}\left(\mathcal{O}_{F} / \mathfrak{N}\right)$ with the stabilizer of $\infty_{\alpha}$ being $\hat{\mathrm{R}}_{\mathfrak{N}, \alpha}^{\times}$, we can rewrite the above bijection as

$$
\begin{aligned}
\mathrm{B}^{\times} \backslash \mathrm{B}^{\times} g_{\alpha} \hat{\mathrm{R}}^{\times} / \hat{\mathrm{R}}_{\mathfrak{N}}^{\times} & \rightarrow \mathrm{R}_{\alpha}^{\times} \backslash \mathbf{P}^{1}\left(\mathcal{O}_{F} / \mathfrak{N}\right), \\
\gamma g_{\alpha} x & \mapsto\left(g_{\alpha} x g_{\alpha}^{-1}\right) \cdot \infty_{\alpha} .
\end{aligned}
$$

It then follows that $f$ uniquely determines the vector $\left(f_{\alpha}\right)_{\alpha}$, and vice versa. Therefore, the map

$$
\begin{aligned}
S_{\underline{k}}^{\mathrm{B}}(\mathfrak{N}) & \rightarrow \bigoplus_{\alpha=1}^{h} S_{\underline{k}}\left(\mathrm{R}_{\alpha}, \mathfrak{N}\right), \\
f & \mapsto\left(f_{\alpha}\right)_{\alpha}
\end{aligned}
$$

is an isomorphism of complex vector spaces, the inverse being obtained as follows. For any $h$-tuple $\left(f_{\alpha}\right)_{\alpha}$, we define $f$ by

$$
f\left(\gamma g_{\alpha} x\right):=f_{\alpha}\left(\left(g_{\alpha} x g_{\alpha}^{-1}\right) \cdot \infty_{\alpha}\right) \gamma^{-1}, \gamma \in G(\mathbb{Q}), x \in \hat{\mathrm{R}}^{\times} .
$$

It remains to prove that this isomorphism is compatible with the Hecke action. To this end, take $u \in \hat{\mathrm{R}}$, with $u \neq 0$, and write the disjoint union

$$
\hat{\mathrm{R}}_{\mathfrak{N}}^{\times} u \hat{\mathrm{R}}_{\mathfrak{N}}^{\times}=\coprod_{\nu} u_{\nu} \hat{\mathrm{R}}_{\mathfrak{N}}^{\times}
$$


Then, the $\alpha$ th component of the form $f$ under the action of the Hecke operator $\left[\hat{\mathrm{R}}_{\mathfrak{N}}^{\times} u \hat{\mathrm{R}}_{\mathfrak{N}}^{\times}\right]$is given by

$$
\begin{aligned}
f \|_{\underline{k}}\left[\hat{\mathrm{R}}_{\mathfrak{N}}^{\times} u \hat{\mathrm{R}}_{\mathfrak{N}}^{\times}\right]_{\alpha}(x) & =\sum_{\nu} f \|_{\underline{k}} u_{\nu}\left(x g_{\alpha}\right)=\sum_{\nu} f\left(x g_{\alpha} u_{\nu}\right) \\
& =\sum_{\beta=1}^{h} \sum_{x g_{\alpha} u_{\nu} \in \mathrm{B}^{\times} g_{\beta} \hat{\mathrm{R}}_{\beta}^{\times}} f\left(x g_{\alpha} u_{\nu}\right) \\
& =\sum_{\beta=1}^{h} \sum_{\nu \in \mathbf{N}\left(x, \alpha, \beta, g_{\alpha} u g_{\beta}^{-1}\right)} f\left(x\left(g_{\alpha} u_{\nu} g_{\beta}^{-1}\right) g_{\beta}\right) \\
& =\sum_{\beta=1}^{h} \sum_{\nu \in \mathbf{N}\left(x, \alpha, \beta, g_{\alpha} u g_{\beta}^{-1}\right)} f_{\beta} \|_{\underline{k}}\left(g_{\alpha} u_{\nu} g_{\beta}^{-1}\right)(x) \\
& =\sum_{\beta=1}^{h} f_{\beta} \|_{\underline{k}}\left[\hat{\mathrm{R}}_{\mathfrak{N}, \alpha}^{\times} g_{\alpha} u g_{\beta}^{-1} \hat{\mathrm{R}}_{\mathfrak{N}, \beta}^{\times}\right](x) .
\end{aligned}
$$

This completes the proof.

From now on, we fix a prime $\mathfrak{q}$ such that $(\mathfrak{q}, \mathfrak{N})=1$. (We simply let $\mathfrak{q}=(1)$, when the class number of the quaternion algebra $B$ is 1.) By making use of the strong approximation theorem, we choose the ideal $I_{\alpha}$ such that $\mathbf{N}\left(I_{\alpha}\right)$ is a power of $\mathfrak{q}$, for each $\alpha=1, \ldots, h$. Now, let $\mathfrak{p} \neq \mathfrak{q}$ be a prime ideal of $F$ such that $(\mathfrak{p}, \mathfrak{N})=1$, and $\pi_{p}$ a uniformizer of $\mathfrak{p}$. For each $\alpha, \beta$, put

$$
\mathbf{N}(\alpha, \beta, \mathfrak{p}):=\left\{u \in I_{\alpha} I_{\beta}^{-1}: \frac{\mathbf{N}(u)}{\mathbf{N}\left(I_{\alpha} I_{\beta}^{-1}\right)}=\pi_{\mathfrak{p}}\right\} / \mathrm{R}_{\alpha}^{\times},
$$

where we let $\mathrm{R}_{\alpha}^{\times}$act by multiplication on the left. The action of Hecke in terms of global elements translates as follows:

$$
\begin{aligned}
T_{\mathfrak{p}}^{\alpha, \beta}: S_{\underline{k}}\left(\mathrm{R}_{\beta}, \mathfrak{N}\right) & \rightarrow S_{\underline{k}}\left(\mathrm{R}_{\alpha}, \mathfrak{N}\right), \\
f & \mapsto \sum_{u \in \mathbf{N}(\alpha, \beta, \mathfrak{p})} f \|_{\underline{k}} u .
\end{aligned}
$$

When applying to an element $x \in \mathbf{P}^{1}\left(\mathcal{O}_{F} / \mathfrak{N}\right)$, the summation must be restricted to the $u$ 's whose action is nondegenerate. The operators $T_{\mathfrak{p}}^{\alpha, \beta}$ generate a (finite) $\mathbb{Z}$-submodule $\mathbf{T}_{k}^{\mathrm{R}_{\alpha}, \mathrm{R}_{\beta}}(\mathfrak{N})$ of $\operatorname{Hom}\left(S_{\underline{k}}\left(\mathrm{R}_{\alpha}, \mathfrak{N}\right), S_{\underline{k}}\left(\mathrm{R}_{\beta}, \mathfrak{N}\right)\right)$. The computation of the action of $\mathbf{T}_{\underline{k}}^{\bar{B}}(\mathfrak{N})$ amounts to the computation of the action of the collection $\left(\mathbf{T}_{k}^{\mathrm{R}_{\alpha}, \mathrm{R}_{\beta}}(\mathfrak{N})\right)$.

We will now describe the Brandt matrices. To this end, let us fix a fundamental domain

$$
S_{\alpha}=\left\{x_{i}^{\alpha}, i=1, \ldots, s_{\alpha}\right\}
$$

for the action of $\mathrm{R}_{\alpha}^{\times}$on $\mathbf{P}^{1}\left(\mathcal{O}_{F} / \mathfrak{N}\right)$, where $s_{\alpha}$ is the cardinality of $S_{\alpha}$. Recalling that each element in $S_{\underline{k}}\left(\mathrm{R}_{\alpha}, \mathfrak{N}\right)$ is completely determined by its values on the set 
$S_{\alpha}$, we get an isomorphism of complex vector spaces

$$
\begin{aligned}
S_{\underline{k}}\left(\mathrm{R}_{\alpha}, \mathfrak{N}\right) & \rightarrow \bigoplus_{i=1}^{s_{\alpha}} L_{\underline{k}}^{\Gamma^{\alpha}}, \\
f & \mapsto\left(f\left(x_{i}^{\alpha}\right)\right)_{i},
\end{aligned}
$$

where $\Gamma_{i}^{\alpha}$ is the stabilizer of $x_{i}^{\alpha}$ and $L_{\underline{k}}^{\Gamma_{i}^{\alpha}}$ is the space of $\Gamma_{i}^{\alpha}$-invariants. To compute the Brandt matrix of $T_{\mathfrak{p}}^{\alpha, \beta}$, we let $\mathbf{N}\left(x_{i}^{\alpha}, x_{j}^{\beta}, \mathfrak{p}\right)$ be the subset of $\mathbf{N}(\alpha, \beta, \mathfrak{p})$ given by

$$
\mathbf{N}\left(x_{i}^{\alpha}, x_{j}^{\beta}, \mathfrak{p}\right)=\left\{u: u \cdot x_{i}^{\alpha}=\gamma_{u} \cdot x_{j}^{\beta} \text { for some } \gamma_{u} \in \mathrm{R}_{\beta}^{\times}\right\} .
$$

Now, take $f \in S_{\underline{k}}\left(\mathrm{R}_{\alpha}, \mathfrak{N}\right)$. Then, we have

$$
\begin{aligned}
f \|_{\underline{k}} T_{\mathfrak{p}}^{\alpha, \beta}\left(x_{i}^{\alpha}\right) & =\sum_{u} f \|_{\underline{k}} u\left(x_{i}^{\alpha}\right)=\sum_{u} f\left(u x_{i}^{\alpha}\right) u \\
& =\sum_{j=1}^{s_{\beta}} \sum_{u \in \mathbf{N}\left(x_{i}^{\alpha}, x_{j}^{\beta}, \mathfrak{p}\right)} f\left(u x_{i}^{\alpha}\right) u \\
& =\sum_{j=1}^{s_{\beta}} \sum_{u \in \mathbf{N}\left(x_{i}^{\alpha}, x_{j}^{\beta}, \mathfrak{p}\right)} f\left(\gamma_{u} x_{j}^{\beta}\right) u \\
& =\sum_{j=1}^{s_{\beta}} f\left(x_{j}^{\beta}\right)\left(\sum_{u \in \mathbf{N}\left(x_{i}^{\alpha}, x_{j}^{\beta}, \mathfrak{p}\right)} \gamma_{u}^{-1} u\right) .
\end{aligned}
$$

So, we define the Brandt matrix of the operator $T_{\mathfrak{p}}^{\alpha, \beta}$ to be $\mathcal{B}_{\mathfrak{p}}^{\alpha, \beta}=\left(b_{i j}\right)$, where the entry $b_{j i}$ is the linear operator

$$
\begin{aligned}
b_{j i}: L_{\underline{k}}^{\Gamma_{j}^{\beta}} & \rightarrow L_{\underline{k}}^{\Gamma_{i}^{\alpha}}, \\
v & \mapsto v\left(\sum_{u \in \mathbf{N}\left(x_{i}^{\alpha}, x_{j}^{\beta}, \mathfrak{p}\right)} \gamma_{u}^{-1} u\right) .
\end{aligned}
$$

Remark 1. One can verify that the definition of the Brandt matrices is independent of the choice of the fundamental domains $S_{\alpha}, 1 \leq \alpha \leq h$.

\section{BRANDT MATRICES With CHARACTER}

To develop the theory of Brandt matrices with character, let us start with the observation that by replacing $U_{0}(\mathfrak{N}) \cong \hat{\mathrm{R}}_{\mathfrak{N}}^{\times}$by $U_{1}(\mathfrak{N})$, the theory we just presented in Sections 2 and 3 will work through, especially, Theorem 2 will still hold, and we will get the following isomorphism of Hecke modules:

$$
S_{\underline{k}}^{\mathrm{B}}\left(U_{1}(\mathfrak{N})\right) \cong \bigoplus_{\alpha=1}^{h} S_{\underline{k}}\left(\mathrm{R}_{\alpha}, U_{1}(\mathfrak{N})\right),
$$

with the obvious definitions. Now, the space $S_{\underline{k}}\left(\mathrm{R}_{\alpha}, U_{1}(\mathfrak{N})\right)$ will just be the set of functions

$$
f: \mathcal{H}_{1}(\mathfrak{N}) \rightarrow L_{\underline{k}} \quad \text { such that } \quad f \|_{\underline{k}} \gamma=f
$$


where

$$
\mathcal{H}_{1}(\mathfrak{N}):=\left\{(a, b) \in(\mathcal{O} / \mathfrak{N})^{2} \text { with } \operatorname{gcd}(a, b) \in(\mathcal{O} / \mathfrak{N})^{\times}\right\} .
$$

The natural action of $(\mathcal{O} / \mathfrak{N})^{\times}$on $\mathcal{H}_{1}(\mathfrak{N})$ induces the following action

$$
(u \cdot f):=f(u x) \quad u \in(\mathcal{O} / \mathfrak{N})^{\times} \text {and } x \in \mathcal{H}_{1}(\mathfrak{N})
$$

on $S_{\underline{k}}\left(\mathrm{R}_{\alpha}, U_{1}(\mathfrak{N})\right)$. We would like to decompose $S_{\underline{k}}\left(\mathrm{R}_{\alpha}, U_{1}(\mathfrak{N})\right)$ under this action. To this end, let us fix a set $S$ of coset representatives for $\mathcal{H}_{1}(\mathfrak{N}) /(\mathcal{O} / \mathfrak{N})^{\times}$. We would like to observe that, though there is a bijection between $S$ and the projective line we considered before, we would like to make a marked distinction between the two. We fix a character $\chi:(\mathcal{O} / \mathfrak{N})^{\times} \rightarrow \mathbb{C}^{\times}$and define the twisted projective line by $\chi$ to be

$$
\mathbf{P}_{\chi}^{1}(\mathcal{O} / \mathfrak{N}):=\mathcal{H}_{1}(\mathfrak{N}) / \operatorname{ker}(\chi)
$$

i.e., $(u a, u b) \sim(a, b) \Longleftrightarrow u \in \operatorname{ker}(\chi)$. Note that, for the trivial character, we get the usual projective line. Also, note that there is a canonical map $\mathbf{P}_{\chi}^{1}(\mathcal{O} / \mathfrak{N}) \rightarrow$ $\mathbf{P}^{1}(\mathcal{O} / \mathfrak{N})$ which is not $\mathrm{R}_{\alpha}^{\times}$-linear in general. We define the space of automorphic forms of level $U_{1}(\mathfrak{N}, \alpha)$, weight $\underline{k}$ and character $\chi$ to be

$$
S_{\underline{k}}\left(\mathrm{R}_{\alpha}, \mathfrak{N}, \chi\right):=\left\{f: \mathbf{P}_{\chi}^{1}(\mathcal{O} / \mathfrak{N}) \rightarrow L_{\underline{k}} \quad \text { such that } f \|_{\underline{k}} \gamma=f\right\} .
$$

Now, take $f \in S_{\underline{k}}\left(\mathrm{R}_{\alpha}, \mathfrak{N}, \chi\right)$ and put

$$
f_{\chi}(u \cdot x):=\chi(u) f(x), \quad u \in(\mathcal{O} / \mathfrak{N})^{\times}, x \in S .
$$

It is not hard to see that $f_{\chi}$ is a well-defined element of $S_{\underline{k}}\left(\mathrm{R}_{\alpha}, U_{1}(\mathfrak{N})\right)$ lying in the $\chi$-eigenspace. Conversely, any $\chi$-eigenvector of the action of $(\mathcal{O} / \mathfrak{N})^{\times}$on $S_{\underline{k}}\left(\mathrm{R}_{\alpha}, U_{1}(\mathfrak{N})\right)$ will determine an element of $S_{\underline{k}}\left(\mathrm{R}_{\alpha}, \mathfrak{N}, \chi\right)$ by the relation above. Thus, we have proved the following result.

Theorem 3. The map

$$
\begin{aligned}
S_{\underline{k}}\left(\mathrm{R}_{\alpha}, U_{1}(\mathfrak{N})\right) & \rightarrow \bigoplus_{\chi} S_{\underline{k}}\left(\mathrm{R}_{\alpha}, \mathfrak{N}, \chi\right), \\
f & \mapsto\left(f_{\chi}\right)_{\chi}
\end{aligned}
$$

is an isomorphism of complex spaces in which the $\chi$-eigenspace corresponds to $S_{\underline{k}}\left(\mathrm{R}_{\alpha}, \mathfrak{N}, \chi\right)$.

By making use of the theorem above, one can develop the theory of Brandt matrices with character mutatis mutandis.

\section{Quaternionic $M$-Symbols}

In this section, we define quaternionic $M$-symbols and give their basic properties. The content of this section was largely inspired by Stein [16], and of course, Merel [11] and Manin [10]. We keep the notations of the previous section, except that we put some integral structure on $L_{\underline{k}}$. We choose a generator $\pi_{\mathfrak{q}}$ of $\mathfrak{q}$ and let $\mathcal{O}_{F,(\mathfrak{q})}=\mathcal{O}_{F}\left[\frac{1}{\pi \mathfrak{q}}\right]$ and $\mathcal{O}_{K,(\mathfrak{q})}=\mathcal{O}_{K,(\mathfrak{q})} \otimes \mathcal{O}_{F,(\mathfrak{q})}$. The integral stucture on $L_{\underline{k}}$ is obtained by letting $L_{\underline{k}}\left(\mathcal{O}_{K,(\mathfrak{q})}\right.$ be the $\mathcal{O}_{K,(\mathfrak{q})}$-submodule of $L_{\underline{k}}$ obtained by replacing $\mathbb{C}$ by $\mathcal{O}_{K(\mathfrak{q})}$ in the definition, where $\mathcal{O}_{K}$ is the ring of integers of $K$. So, for each $\mathcal{O}_{K,(\mathfrak{q})}$-algebra $A$, we now define

$$
L_{\underline{k}}(A)=\bigotimes_{\tau \in I} \mathbf{S}_{m_{\tau, v_{\tau}}}(A),
$$


with the right action now restricted to $\mathrm{M}_{2}(A)^{I}$. We fix an isomorphism $j: \mathrm{B} \otimes K \cong$ $\mathrm{M}_{2}(K)^{I}$ such that $j\left(\mathrm{R} \otimes \mathcal{O}_{K}\right)=\mathrm{M}_{2}\left(\mathcal{O}_{K}\right)^{I}$.

We consider the product of $\mathcal{O}_{K,(\mathfrak{q}) \text {-modules }}$

$$
\mathcal{O}_{K,(\mathfrak{q})}\left[\mathbf{P}^{1}(\mathcal{O} / \mathfrak{N})\right] \times L_{\underline{k}}\left(\mathcal{O}_{K,(\mathfrak{q})}\right)
$$

and define a left action of $\mathrm{R}^{\times}$on it by

$$
\gamma \cdot(x, v):=\left(\gamma x, v \gamma^{-1}\right) .
$$

We let $\mathcal{M}_{\underline{k}}\left(\mathrm{R}, \mathfrak{N}, \mathcal{O}_{K,(\mathfrak{q})}\right)$ be the largest torsion-free quotient

$$
\mathcal{O}_{K,(\mathfrak{q})}\left[\mathbf{P}^{1}(\mathcal{O} / \mathfrak{N})\right] \otimes L_{\underline{k}}\left(\mathcal{O}_{K,(\mathfrak{q})}\right) /\left\langle x-\gamma x, \gamma \in \mathrm{R}^{\times}\right\rangle .
$$

We see from that definition that replacing $\mathrm{R}$ by a conjugate order will give an isomorphic $\mathcal{O}_{K,(\mathfrak{q})}$-module. So, we can make the following definition.

Definition 4. We call $\mathcal{M}_{\underline{k}}\left(\mathrm{R}, \mathfrak{N}, \mathcal{O}_{K,(\mathfrak{q})}\right)$ the $\mathcal{O}_{K,(\mathfrak{q})}$-module of quaternionic $M$ symbols of weight $\underline{k}$ and level $\mathfrak{N}$ attached to R.

With obvious definitions, we see that for any subring $\mathcal{O}_{K,(\mathfrak{q})} \subset A \subset \mathbb{C}$,

$$
\mathcal{M}_{\underline{k}}(\mathrm{R}, \mathfrak{N}, A)=\mathcal{M}_{\underline{k}}\left(\mathrm{R}, \mathfrak{N}, \mathcal{O}_{K,(\mathfrak{q})}\right) \otimes_{\mathcal{O}_{K,(\mathfrak{q})}} A .
$$

Let $m=\sum_{i} x_{i} \otimes v_{i}$ be an element of $\mathcal{M}_{\underline{k}}(\mathrm{R}, \mathfrak{N}, \mathbb{C})$, with $x_{i} \in \mathbf{P}^{1}(\mathcal{O} / \mathfrak{N})$ and $v_{i} \in L_{\underline{k}}(\mathbb{C})$. We define the support of $m$ to be

$$
\operatorname{Supp}(m)=\bigcup_{i} \mathrm{R}^{\times} x_{i}
$$

Then, we define the map

$$
\begin{aligned}
f_{m}: \mathbf{P}^{1}(\mathcal{O} / \mathfrak{N}) & \rightarrow \begin{cases}v_{i} \gamma_{i}^{-1}, & x=\gamma_{i} x_{i} \in \operatorname{Supp}(m), \\
0, & \text { else. }\end{cases}
\end{aligned}
$$

It is not hard to see that $f_{m}$ is an element of $S_{\underline{k}}(\mathrm{R}, \mathfrak{N})$.

\section{Proposition 4.}

$$
\begin{aligned}
\mathcal{M}_{\underline{k}}\left(\mathrm{R}, \mathfrak{N}, \mathcal{O}_{K,(\mathfrak{q})}\right) & \rightarrow S_{\underline{k}}\left(\mathrm{R}, \mathfrak{N}, \mathcal{O}_{K,(\mathfrak{q})}\right), \\
m & \mapsto f_{m}
\end{aligned}
$$

is an isomorphism of $\mathcal{O}_{K,(\mathfrak{q})}$-modules.

Proof. We only need show that

$$
\begin{aligned}
\mathcal{M}_{\underline{k}}(\mathrm{R}, \mathfrak{N}, \mathbb{C}) & \rightarrow S_{\underline{k}}(\mathrm{R}, \mathfrak{N}), \\
m & \mapsto f_{m}
\end{aligned}
$$

is an isomorphism of complex spaces, but this is not very hard to see from the definition.

Definition 5. The $\mathcal{O}_{K,(\mathfrak{q})}$-module of quaternionic $M$-symbols of level $\mathfrak{N}$ and weight $\underline{k}$ attached to $\mathrm{B}$ is defined by

$$
\mathcal{M}_{\underline{k}}^{\mathrm{B}}\left(\mathfrak{N}, \mathcal{O}_{K,(\mathfrak{q})}\right):=\bigoplus_{\alpha=1}^{h} \mathcal{M}_{\underline{k}}\left(\mathrm{R}_{\alpha}, \mathfrak{N}, \mathcal{O}_{K,(\mathfrak{q})}\right) .
$$


We now define the action of Hecke on this module by

$$
\begin{aligned}
T_{\mathfrak{p}}^{\alpha, \beta}: \mathcal{M}_{\underline{k}}\left(\mathrm{R}_{\alpha}, \mathfrak{N}, \mathcal{O}_{K,(\mathfrak{q})}\right) & \rightarrow \mathcal{M}_{\underline{k}}\left(\mathrm{R}_{\beta}, \mathfrak{N}, \mathcal{O}_{K,(\mathfrak{q})}\right), \\
{[x]_{\alpha} } & \mapsto \sum_{u \in \mathbf{N}(\alpha, \beta, \mathfrak{p})}[u \cdot x]_{\beta},
\end{aligned}
$$

where $[x]_{\alpha}$ denotes the class of $x \in \mathcal{O}_{K,(\mathfrak{q})}\left[\mathbf{P}^{1}(\mathcal{O} / \mathfrak{N})\right] \otimes L_{\underline{k}}\left(\mathcal{O}_{K,(\mathfrak{q})}\right)$ in the module $\mathcal{M}_{\underline{k}}\left(\mathrm{R}_{\alpha}, \mathfrak{N}, \mathcal{O}_{K,(\mathfrak{q})}\right)$. We get the Hecke operator $T_{\mathfrak{p}}$ on $\mathcal{M}_{\underline{k}}^{\mathrm{B}}\left(\mathfrak{N}, \mathcal{O}_{K}\right)$ by piecing together all the $T_{\mathfrak{p}}^{\alpha, \beta}$, s.

Theorem 5. The map

$$
\begin{aligned}
\mathcal{M}_{\underline{k}}^{\mathrm{B}}\left(\mathfrak{N}, \mathcal{O}_{K,(\mathfrak{q})}\right) & \rightarrow S_{\underline{k}}^{\mathrm{B}}\left(\mathfrak{N}, \mathcal{O}_{K,(\mathfrak{q})}\right), \\
\left(m_{\alpha}\right)_{\alpha} & \mapsto\left(f_{m_{\alpha}}\right)_{\alpha}
\end{aligned}
$$

is an isomorphism of Hecke modules.

Proof. This is a restatement of Theorem 2 in light of Proposition 4.

Remark 2. 1) The analogy between our quaternionic $M$-symbols and the Manin symbols, for $F=\mathbb{Q}$, is clearly transparent from the definition. Further, the same way the Manin symbols give a purely algebraic description of the holomorphic cuspidal cohomology of the modular curve which lends itself very well to computation, so do our quaternionic $M$-symbols. In our case however, one needs to resort to the Jacquet-Langlands correspondence in order to prove that the holomorphic cuspidal part of the cohomology of the Hilbert modular variety $X_{0}(\mathfrak{N})$ is given by our quaternionic $M$-symbols, whereas in the rational case, one disposes of the so-called Manin trick. One of our goals is to implement the computation of the module of quaternionic $M$-symbols into a package similar to the Magma package of Stein [9] for $M$-symbols, at least for a wide range of quadratic fields.

2) From a computational point of view, the module $\mathcal{M}_{\underline{k}}^{\mathrm{B}}\left(\mathfrak{N}, \mathcal{O}_{K,(\mathfrak{q})}\right)$ can also be used in approaching algebraic modular forms as defined in Gross [7. Indeed, let $G / \mathbb{Q}$ be a reductive group satisfying the conditions in [7, Proposition 1.4], and $U \subset G(\hat{\mathbb{Z}})$ a compact open subgroup. In most interesting cases, the quotient $G(\hat{\mathbb{Z}}) / U$ will often have a nice description as a flag variety over a finite artinian ring. So, knowing the class number of $G$, one can give a description of the space of algebraic modular forms of level $U$ similar to ours, and get the Hecke operators as acting on a disjoint union of such flag varieties. In fact, a similar approach was adopted by Pollack in his thesis [13] in order to compute coset representatives for $G(\mathbb{Q}) \backslash G\left(\mathbb{A}_{f}\right) / U$, except that for the description of Hecke action, he went back to the adelic setting.

\section{Algorithms For ReAl QUADRATIC FieldS}

We will now explain how the discussion in the previous sections can be used for explicit computation of Hilbert modular forms on real quadratic fields. In order to do so we need some preliminary results. We assume for the rest of this section that $F=\mathbb{Q}(\sqrt{D})$ is a real quadratic field of narrow class number one, where $D$ is a square-free positive integer. Let $v_{1}$ and $v_{2}$ be the two real places of $F$. We assume that $F$ has a fundamental unit $\varepsilon$ with $v_{1}(\varepsilon)>0, v_{2}(\varepsilon)<0$ and $\mathbf{N}(\varepsilon)=-1$. 
6.1. Choosing the definite quaternion algebra. To start our algorithm, we need a totally definite quaternion algebra $\mathrm{B}$ of center $F$ with $\operatorname{Ram}(\mathrm{B})=\left\{v_{1}, v_{2}\right\}$. The following lemma will help in that choice.

Lemma 1. Let $p$ be a positive prime in $\mathbb{Q}$ that is inert or ramified in $F$, and $\mathrm{B}$ the quaternion algebra

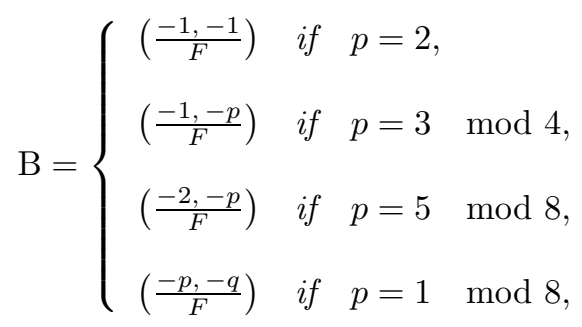

where $q$ is a positive prime in $\mathbb{Q}$ with $q=3 \bmod 4$ and $\left(\frac{p}{q}\right)=-1$. Then $\mathrm{B}$ is the unique (up to isomorphism) totally definite quaternion algebra with $\operatorname{Ram}(\mathrm{B})=$ $\left\{v_{1}, v_{2}\right\}$.

Proof. Let $\mathrm{B}_{\infty, p}$ be the unique (up to isomorphism) quaternion algebra defined over $\mathbb{Q}$ which is ramified only at $\infty$ and $p$. By Pizer [12, Proposition 5.1], there is a canonical embedding of $\mathrm{B}_{\infty, p}$ into $\mathrm{B}$. Therefore, $\mathrm{B}$ can only ramify at primes that lie above $\infty$ and $p$. But $\mathrm{B}$ clearly ramifies above the two primes $v_{1}$ and $v_{2}$. And, since $p$ is inert or ramified in $F$ and $\operatorname{Ram}(\mathrm{B})$ must have even cardinality, we see that $\operatorname{Ram}(\mathrm{B})$ can only consist of $\left\{v_{1}, v_{2}\right\}$.

Remark 3. To kick start our algorithm, we need to exhibit a maximal order $\mathrm{R}$ in B. There is a result in Socrates and Whitehouse [15] which does this. The proof which is valid only for quadratic field with discriminant $D \equiv 5(8)$ is essentially computational in nature. One can always write an easy algorithm which searches for the basis of a maximal order. As a starting point, if we need a quaternion algebra $\mathrm{B}$ in which the prime $p$ is inert, we can take the basis of the quaternion algebra $\mathrm{B}_{\infty, p}$ given by Pizer [12, Proposition 5.2], and look for a maximal order in $\mathrm{B}$ such that the transition matrix to its basis is in Hermite normal form. Then, we only need to do an efficient search for coefficients in the finite field $\mathbb{F}_{p^{2}}$. We see that one needs to choose $p$ as small as possible.

6.2. Computing representatives for the (right) R-ideal classes. Once we have chosen the quaternion algebra $\mathrm{B}$ and the maximal order $\mathrm{R}$, the steps of the algorithm follow more or less the ones in Pizer [12] and Consani and Scholten [1]. We recall some of the results in those two papers, which can be very useful.

Proposition 6. Let $I$ and $J$ be two right R-ideals. Then, $I$ and $J$ belong to the same class if and only if there is an element $\gamma \in I \bar{J}$ such that $\mathbf{N}(\gamma)=\mathbf{N}(I) \mathbf{N}(J)$.

Proof. See Pizer [12, Proposition 1.18], and Consani and Scholten [1, Proposition 8.1].

Remark 4. We can use this proposition to search for ideals in the form $I=\mathfrak{a R}$, where $\mathfrak{a}$ is an ideal in some quadratic extension $K=F[\alpha]$, where $\alpha \in \mathrm{B}-F$. That we can always find representatives of ideal classes in this form follows from [15, Theorem 7.2]. The search can be made more efficient by first comparing the theta series of the ideals. This is discussed at length in [15, Chap. 7], see also [1, 12]. 
6.3. Computing the Brandt matrices. The computation of the global elements in $I_{\alpha, \beta}$ necessary for the calculation of the Hecke operators relies on the efficiency of the algorithm that represents an element of $F$ by the quadratic form that gives the scaled norm of an element in B. Again, we refer to Pizer [12, sec. 6] and Consani and Scholten [1, sec. 8], and Socrates and Whitehouse [15, Chap. 6 and 7]. We only add that there are now many procedures in Magma which facilitate working with lattices (see the pages on lattices in 9]).

6.4. Computing the local embeddings. The computation of the local embeddings is fairly easy. This amounts to finding basis elements in $\mathrm{M}_{2}(\mathcal{O} / \mathfrak{N})$ satisfying the same relations as the ones by the elements in the basis of the corresponding maximal order. Most of time, the denominators of the basis elements are units in $\mathcal{O} / \mathfrak{N}$, so we can just take a basis of $\mathrm{M}_{2}(\mathcal{O} / \mathfrak{N})$ satisfying the same relations as $i, j$. Otherwise, we need to do the same work starting with a basis of the maximal order.

Remark 5. The implementation of the algorithm proceeds as in 44. The main difference between our algorithm and the modular symbol algorithm for classical modular forms resides in Step 1 which is not needed when working with the matrix algebra $M_{2}(\mathbb{Q})$. The computations in Step 1 can, however, be performed and the results stored in a database for each quadratic field. Our algorithm then becomes as efficient as the modular symbols one. One of our goals is to create a huge database of quadratic fields that can be used in a package similar to the Hecke package of Stein [16] in Magma.

\section{NUMERICAL EXAMPLES}

In this section, we present two numerical examples on real quadratic fields. We think that the algorithm given here should be easy to rewrite for any totally real number field of narrow class number one. In the computations of this section, the results in Socrates and Whitehouse [15] were very helpful, especially the one relating the type number and the class number of certain quaternion algebras over real quadratic fields. We have chosen two quadratic fields with the prime 2 inert in them. So, the unique (up to isomorphism) totally definite quaternion algebra ramified only at both infinite places is the Hamilton quaternion algebra B. We consider its canonical basis $\{1, i, j, k\}$, with $i^{2}=j^{2}=-1, k=i j=-j i$. For any quadratic field $F$ of discriminant $D$, we will let $\omega_{D}$ be a generator of its maximal $\operatorname{order} \mathcal{O}_{F}$.

7.1. $F=\mathbb{Q}(\sqrt{29})$. From Socrates and Whitehouse [15], we know that B has class number $h=2$. We chose the maximal order whose basis is given in Socrates and Whitehouse:

$$
\mathrm{R}:=\left\langle\frac{1+i+j+k}{2}, \frac{i+\omega_{29} j+\left(1+\omega_{29}\right) k}{2}, j, k\right\rangle .
$$

The two representatives of the ideals classes we chose were

$$
I_{1}:=\mathrm{R} \quad \text { and } \quad I_{2}:=\left(9+4 \omega_{29}, 19+\omega_{29}+6 i\right) \mathrm{R} .
$$

See Table 1 for the list of all newforms of level a prime of norm less than 100 and whose coefficients are rational or defined over a quadratic field. The levels and the forms are listed up to Galois conjugation. 
TABLE 1. Hilbert modular forms of weight $(2,2)$ on $F=\mathbb{Q}(\sqrt{29})$.

\begin{tabular}{|c|c|c|c|c|c|}
\hline \multicolumn{2}{|c|}{$\mathfrak{N}$} & 1 & $(4,2)$ & $\left(7,0+1 \omega_{29}\right)$ & $(9,3)$ \\
\hline $\mathbf{N}(\mathfrak{p})$ & $\mathfrak{p}$ & $a\left(\mathfrak{p}, f_{1}\right)$ & $a\left(\mathfrak{p}, f_{1}\right)$ & $a\left(\mathfrak{p}, f_{1}\right)$ & $a\left(\mathfrak{p}, f_{1}\right)$ \\
\hline \hline 4 & 2 & -1 & -1 & -1 & $3-1 \omega_{5}$ \\
9 & 3 & 1 & 5 & -4 & -1 \\
5 & $1+1 \omega_{29}$ & -3 & 1 & 2 & $0+2 \omega_{5}$ \\
5 & $3+1 \omega_{29}$ & -3 & 1 & 2 & $0+2 \omega_{5}$ \\
7 & $7+3 \omega_{29}$ & 2 & -2 & -1 & $-1-2 \omega_{5}$ \\
7 & $5+2 \omega_{29}$ & 2 & -2 & 2 & $-1-2 \omega_{5}$ \\
13 & $4+1 \omega_{29}$ & -1 & -1 & -6 & -1 \\
13 & $5-1 \omega_{29}$ & -1 & -1 & 4 & -1 \\
\hline
\end{tabular}

\begin{tabular}{|c|c|c|c|c|c|}
\hline \multicolumn{2}{|c|}{$\mathfrak{N}$} & $\left(13,5-1 \omega_{29}\right)$ & \multicolumn{2}{c|}{$\left(29,-1+2 \omega_{29}\right)$} & $\left(53,-2-3 \omega_{29}\right)$ \\
\hline $\mathbf{N}(\mathfrak{p})$ & $\mathfrak{p}$ & $a\left(\mathfrak{p}, f_{1}\right)$ & $a\left(\mathfrak{p}, f_{1}\right)$ & $a\left(\mathfrak{p}, f_{2}\right)$ & $a\left(\mathfrak{p}, f_{1}\right)$ \\
\hline \hline 4 & 2 & -1 & $-1-2 \omega_{8}$ & 0 & $-2+2 \omega_{5}$ \\
9 & 3 & -4 & $-3-2 \omega_{8}$ & 0 & $2-2 \omega_{5}$ \\
5 & $1+1 \omega_{29}$ & -3 & -1 & $0+2 \omega_{5}$ & $0-1 \omega_{5}$ \\
5 & $3+1 \omega_{29}$ & 2 & -1 & $2-2 \omega_{5}$ & 2 \\
7 & $7+3 \omega_{29}$ & -3 & $0+2 \omega_{8}$ & $0+1 \omega_{5}$ & 2 \\
7 & $5+2 \omega_{29}$ & -3 & $0+2 \omega_{8}$ & $1-1 \omega_{5}$ & 2 \\
13 & $4+1 \omega_{29}$ & 4 & $-1+2 \omega_{8}$ & 4 & 4 \\
13 & $5-1 \omega_{29}$ & 1 & $-1+2 \omega_{8}$ & 4 & $-2+2 \omega_{5}$ \\
\hline
\end{tabular}

\begin{tabular}{|c|c|c|c|c|c|}
\hline \multicolumn{2}{|c|}{$\mathfrak{N}$} & \multicolumn{2}{c|}{$\left(59,-4+3 \omega_{29}\right)$} & \multicolumn{2}{c|}{$\left(67,-10-3 \omega_{29}\right)$} \\
\hline $\mathbf{N}(\mathfrak{p})$ & $\mathfrak{p}$ & $a\left(\mathfrak{p}, f_{1}\right)$ & $a\left(\mathfrak{p}, f_{2}\right)$ & $a\left(\mathfrak{p}, f_{1}\right)$ & $a\left(\mathfrak{p}, f_{2}\right)$ \\
\hline \hline 4 & 2 & -1 & $1+1 \omega_{8}$ & -3 & $0+1 \omega_{5}$ \\
9 & 3 & 5 & 4 & 5 & $-1-2 \omega_{5}$ \\
5 & $1+1 \omega_{29}$ & 0 & $0-2 \omega_{8}$ & -1 & -1 \\
5 & $3+1 \omega_{29}$ & 0 & 2 & -3 & $2-2 \omega_{5}$ \\
7 & $7+3 \omega_{29}$ & -1 & $3-1 \omega_{8}$ & -4 & $-1+1 \omega_{5}$ \\
7 & $5+2 \omega_{29}$ & 5 & $0+2 \omega_{8}$ & 2 & $-1-1 \omega_{5}$ \\
13 & $4+1 \omega_{29}$ & -1 & $-4+2 \omega_{8}$ & -3 & $-5+3 \omega_{5}$ \\
13 & $5-1 \omega_{29}$ & -4 & $-3-1 \omega_{8}$ & 7 & $-3+4 \omega_{5}$ \\
\hline
\end{tabular}

\begin{tabular}{|c|c|c|c|c|}
\hline \multicolumn{2}{|c|}{$\mathfrak{N}$} & $\left(67,-10-3 \omega_{29}\right)$ & \multicolumn{2}{c|}{$\left(71,-9-2 \omega_{29}\right)$} \\
\hline $\mathbf{N}(\mathfrak{p})$ & $\mathfrak{p}$ & $a\left(\mathfrak{p}, f_{3}\right)$ & $a\left(\mathfrak{p}, f_{1}\right)$ & $a\left(\mathfrak{p}, f_{2}\right)$ \\
\hline \hline 4 & 2 & $-2+2 \omega_{5}$ & 3 & $-1+1 \omega_{5}$ \\
9 & 3 & $0+2 \omega_{5}$ & 1 & -3 \\
5 & $1+1 \omega_{29}$ & 2 & 1 & 3 \\
5 & $3+1 \omega_{29}$ & $0-1 \omega_{5}$ & 1 & $-1+1 \omega_{5}$ \\
7 & $7+3 \omega_{29}$ & $0-1 \omega_{5}$ & -2 & $-1+3 \omega_{5}$ \\
7 & $5+2 \omega_{29}$ & $-2-2 \omega_{5}$ & 2 & $0+2 \omega_{5}$ \\
13 & $4+1 \omega_{29}$ & $-2-3 \omega_{5}$ & 3 & $2-4 \omega_{5}$ \\
13 & $5-1 \omega_{29}$ & $-2+2 \omega_{5}$ & -1 & $1+1 \omega_{5}$ \\
\hline
\end{tabular}


7.2. $F=\mathbb{Q}(\sqrt{37})$. Again, from Socrates and Whitehouse [15], we know that B has class number $h=2$. The chosen maximal order was

$$
\mathrm{R}:=\left\langle\frac{1+i+j+k}{2}, \frac{i+\omega_{37} j+\left(1+\omega_{37}\right) k}{2}, j, k\right\rangle .
$$

The two representatives of the ideals classes we chose were

$$
I_{1}:=\mathrm{R} \quad \text { and } \quad I_{2}:=\left(3+\omega_{37}, 2-\frac{1+i+j+k}{2}\right) \mathrm{R} .
$$

See Table 2 for the list of all newforms of level a prime of norm less than 100 and whose coefficients are rational or defined over a quadratic field. The levels and the forms are listed up to Galois conjugation.

TABLE 2. Hilbert modular forms of weight $(2,2)$ on $F=\mathbb{Q}(\sqrt{37})$.

\begin{tabular}{|c|c|c|c|c|c|}
\hline \multicolumn{2}{|c|}{$\mathfrak{N}$} & 1 & $(4,2)$ & \multicolumn{2}{c|}{$\left(11,5-1 \omega_{37}\right)$} \\
\hline $\mathbf{N}(\mathfrak{p})$ & $\mathfrak{p}$ & $a\left(\mathfrak{p}, f_{1}\right)$ & $a\left(\mathfrak{p}, f_{1}\right)$ & $a\left(\mathfrak{p}, f_{1}\right)$ & $a\left(\mathfrak{p}, f_{2}\right)$ \\
\hline \hline 4 & 2 & 0 & -1 & 1 & 3 \\
3 & $3+1 \omega_{37}$ & -1 & $-1+1 \omega_{21}$ & 1 & -1 \\
3 & $4-1 \omega_{37}$ & -1 & $-1+1 \omega_{21}$ & 2 & 2 \\
25 & 5 & 6 & $1+3 \omega_{21}$ & -1 & -9 \\
7 & $11-3 \omega_{37}$ & 3 & -2 & 3 & -3 \\
7 & $8+3 \omega_{37}$ & 3 & -2 & -5 & 3 \\
11 & $4+1 \omega_{37}$ & -3 & $2-1 \omega_{21}$ & 4 & 0 \\
11 & $5-1 \omega_{37}$ & -3 & $2-1 \omega_{21}$ & -1 & -1 \\
\hline
\end{tabular}

\begin{tabular}{|c|c|c|c|c|c|}
\hline \multicolumn{2}{|c|}{$\mathfrak{N}$} & \multicolumn{4}{c|}{$(25,5)$} \\
\hline $\mathbf{N}(\mathfrak{p})$ & $\mathfrak{p}$ & $a\left(\mathfrak{p}, f_{1}\right)$ & $a\left(\mathfrak{p}, f_{2}\right)$ & $a\left(\mathfrak{p}, f_{3}\right)$ & $a\left(\mathfrak{p}, f_{4}\right)$ \\
\hline \hline 4 & 2 & 0 & 0 & 4 & -1 \\
3 & $3+1 \omega_{37}$ & -3 & 1 & 1 & $0+2 \omega_{5}$ \\
3 & $4-1 \omega_{37}$ & 1 & -3 & 1 & $2-2 \omega_{5}$ \\
25 & 5 & 1 & 1 & -1 & -1 \\
7 & $11-3 \omega_{37}$ & 1 & -3 & 1 & $0+2 \omega_{5}$ \\
7 & $8+3 \omega_{37}$ & -3 & 1 & 1 & $2-2 \omega_{5}$ \\
11 & $4+1 \omega_{37}$ & 1 & 1 & -3 & $4-4 \omega_{5}$ \\
11 & $5-1 \omega_{37}$ & 1 & 1 & -3 & $0+4 \omega_{5}$ \\
\hline
\end{tabular}

\begin{tabular}{|c|c|c|c|c|c|}
\hline \multicolumn{2}{|c|}{$\mathfrak{N}$} & \multicolumn{3}{|c|}{$\left(37,-1+2 \omega_{37}\right)$} & $\left(41,5+3 \omega_{37}\right)$ \\
\hline $\mathbf{N}(\mathfrak{p})$ & $\mathfrak{p}$ & $a\left(\mathfrak{p}, f_{1}\right)$ & $a\left(\mathfrak{p}, f_{2}\right)$ & $a\left(\mathfrak{p}, f_{3}\right)$ & $a\left(\mathfrak{p}, f_{1}\right)$ \\
\hline \hline 4 & 2 & -4 & 0 & 0 & -3 \\
3 & $3+1 \omega_{37}$ & 1 & -3 & 2 & -1 \\
3 & $4-1 \omega_{37}$ & 1 & -3 & 2 & -1 \\
25 & 5 & -10 & -6 & -6 & -3 \\
7 & $11-3 \omega_{37}$ & -1 & -1 & $3-3 \omega_{5}$ & 0 \\
7 & $8+3 \omega_{37}$ & -1 & -1 & $0+3 \omega_{5}$ & 0 \\
11 & $4+1 \omega_{37}$ & 3 & -5 & 0 & 6 \\
11 & $5-1 \omega_{37}$ & 3 & -5 & 0 & 0 \\
\hline
\end{tabular}




\begin{tabular}{|c|c|c|c|c|c|}
\hline \multicolumn{2}{|c|}{$\mathfrak{N}$} & \multicolumn{3}{|c|}{$\left(53,-7+3 \omega_{37}\right)$} & $\left(67,-11+4 \omega_{37}\right)$ \\
\hline $\mathbf{N}(\mathfrak{p})$ & $\mathfrak{p}$ & $a\left(\mathfrak{p}, f_{1}\right)$ & $a\left(\mathfrak{p}, f_{2}\right)$ & $a\left(\mathfrak{p}, f_{3}\right)$ & $a\left(\mathfrak{p}, f_{1}\right)$ \\
\hline \hline 4 & 2 & 1 & -3 & 2 & 0 \\
3 & $3+1 \omega_{37}$ & 2 & 2 & 2 & -1 \\
3 & $4-1 \omega_{37}$ & -1 & -1 & $2-1 \omega_{5}$ & 1 \\
25 & 5 & -2 & 6 & $-2+6 \omega_{5}$ & 2 \\
7 & $11-3 \omega_{37}$ & 5 & -3 & $0-1 \omega_{5}$ & -3 \\
7 & $8+3 \omega_{37}$ & 0 & 0 & $2+1 \omega_{5}$ & -1 \\
11 & $4+1 \omega_{37}$ & 5 & -3 & $0+4 \omega_{5}$ & 3 \\
11 & $5-1 \omega_{37}$ & 3 & 3 & $-5+1 \omega_{5}$ & 5 \\
\hline
\end{tabular}

\begin{tabular}{|c|c|c|c|c|c|}
\hline \multicolumn{2}{|c|}{$\mathfrak{N}$} & \multicolumn{2}{|c|}{$\left(71,2+3 \omega_{37}\right)$} & $\left(73,14-3 \omega_{37}\right)$ & $\left(83,1-3 \omega_{37}\right)$ \\
\hline $\mathbf{N}(\mathfrak{p})$ & $\mathfrak{p}$ & $a\left(\mathfrak{p}, f_{1}\right)$ & $a\left(\mathfrak{p}, f_{2}\right)$ & $a\left(\mathfrak{p}, f_{1}\right)$ & $a\left(\mathfrak{p}, f_{1}\right)$ \\
\hline \hline 4 & 2 & -2 & -1 & -2 & $-1+1 \omega_{5}$ \\
3 & $3+1 \omega_{37}$ & 0 & 0 & -3 & -1 \\
3 & $4-1 \omega_{37}$ & 2 & 2 & 3 & $1+1 \omega_{5}$ \\
25 & 5 & -3 & 6 & 4 & $-4+6 \omega_{5}$ \\
7 & $11-3 \omega_{37}$ & -4 & 4 & 0 & 2 \\
7 & $8+3 \omega_{37}$ & 1 & -4 & 4 & $-4+1 \omega_{5}$ \\
11 & $4+1 \omega_{37}$ & 5 & 4 & 4 & $-1+4 \omega_{5}$ \\
11 & $5-1 \omega_{37}$ & 0 & 6 & 3 & $2+1 \omega_{5}$ \\
\hline
\end{tabular}

\begin{tabular}{|c|c|c|}
\hline \multicolumn{2}{|c|}{$\mathfrak{N}$} & $\left(83,1-3 \omega_{37}\right)$ \\
\hline $\mathbf{N}(\mathfrak{p})$ & $\mathfrak{p}$ & $a\left(\mathfrak{p}, f_{2}\right)$ \\
\hline \hline 4 & 2 & $-3+1 \omega_{5}$ \\
3 & $3+1 \omega_{37}$ & -1 \\
3 & $4-1 \omega_{37}$ & $1+1 \omega_{5}$ \\
25 & 5 & $4-6 \omega_{5}$ \\
7 & $11-3 \omega_{37}$ & -2 \\
7 & $8+3 \omega_{37}$ & $0+1 \omega_{5}$ \\
11 & $4+1 \omega_{37}$ & $3-2 \omega_{5}$ \\
11 & $5-1 \omega_{37}$ & $0-1 \omega_{5}$ \\
\hline
\end{tabular}

Remark 6 . One very amusing and pleasant thing which confirmed that our computations were correct was the level one forms appearing in all subsequent levels as oldforms. Since, those forms can be found elsewhere, this was a proof that the algorithm was working. Indeed, the level one forms correspond to elliptic curves with everywhere good reduction, and they can be found in several places (see, for example, Darmon and Logan [2]). We give them below; $E_{29}$ is the one defined over $\mathbb{Q}(\sqrt{29})$ and $E_{37}$ is the one defined over $\mathbb{Q} \sqrt{37}$.

$$
E_{29}: y^{2}+x y+\left(2+\omega_{29}\right)^{2} y=x^{3}
$$

and

$$
E_{37}: y^{2}+y=x^{3}+2 x^{2}-\left(19+8 \omega_{37}\right) x+\left(28+11 \omega_{37}\right) .
$$

Remark 7. Our final remark is that it could be better to implement a variant of this algorithm if we know more about the cuspidal representation associated to the form we are looking for. For example, if we know that this representation is special 
or supercuspidal at more than two primes, say $\mathfrak{p}$ and $\mathfrak{q}$, we could choose instead the quaternion algebra $B$ to be ramified at both places at infinity and the two primes $\mathfrak{p}$ and $\mathfrak{q}$. This will have the tremendous advantage of cutting down the dimension of the space we are looking for. This trick could have been used in [1] for example. In the same way, one can implement a variant of this algorithm over field of odd degree by adding an extra prime to the ramification of the quaternion algebra. Using the Hamilton quaternion algebra over $\mathbb{Q}$, for example, would give all forms that are new at 2. This can be seen as a variant of Pizer [12].

\section{ACKNOWLEDGMENTS}

This paper was started while I was a post-doctoral fellow at Brandeis University, and finished at the University of Calgary where I am currently holding a postdoctoral position as well. I would like to thank both institutions for their hospitality and their support, especially Professor Fred Diamond at Brandeis University, and Professor Hugh Williams at University of Calgary. I would also like to thank Professor Henri Darmon for his careful reading of an earlier version of this paper. His suggestions helped a lot to improve the presentation in this final version. My final thanks go to the anonymous referee.

\section{REFERENCES}

[1] C. Consani and J. Scholten, Arithmetic on a quintic threefold. International Journal of Mathematics 12, No. 8 (2001), pp. 943-972. MR.1863287(2002h:11058)

[2] Henri Darmon and Adam Logan, Periods of Hilbert modular forms and rational points on elliptic curves. Int. Math. Res. Not. 2003, no. 40, 2153-2180. MR1997296 (2005f:11110)

[3] L. Dembélé, Explicit computations of Hilbert modular forms on $\mathbb{Q}(\sqrt{5})$. Ph.D. Thesis at McGill University, 2002.

[4] L. Dembélé, Explicit computations of Hilbert modular forms on $\mathbb{Q}(\sqrt{5})$. Experiment. Math. 14 (2005), no. 4, 457-466. MR2193808 (2006h:11050)

[5] L. Dembélé, F. Diamond and Robert; Numerical evidences of the weight part of the Serre conjecture for Hilbert modular forms. (preprint).

[6] Stephen S. Gelbart, Automorphic forms on adèle groups. Annals of Mathematics Studies, No. 83. Princeton University Press, Princeton, N.J.; University of Tokyo Press, Tokyo, 1975. MR0379375 (52:280)

[7] Benedict H. Gross, Algebraic modular forms. Israel J. Math. 113 (1999), 61-93. MR1729443 (2001b:11037)

[8] H. Jacquet and R. P. Langlands, Automorphic forms on GL(2). Lecture Notes in Mathematics, Vol. 114. Springer-Verlag, Berlin-New York, 1970. MR0401654 (53:5481)

[9] Magma algebraic computing system. http://magma.maths.usyd.edu.au

[10] Ju. I. Manin, Parabolic points and zeta functions of modular curves. Math. USSR-Izv. 6 (1972), 19-64. MR0314846 (47:3396)

[11] L. Merel, Universal Fourier expansion of modular forms. Lecture Notes in Math. 1585. Springer-Verlag, 1994. MR1322319 (96h:11032)

[12] A. Pizer, An algorithm for computing modular forms on $\Gamma_{0}(N)$. Journal of Algebra 64, (1980), pp.340-390. MR.0579066 (83g:10020)

[13] D. Pollack, Explicit Hecke action on modular forms. Ph.D. thesis at Havard University 1998.

[14] G. Shimura, The special values of the zeta functions associated to Hilbert modular forms. Duke Math. J. 45, No. 3 (1978), pp. 637-679. MR0507462 (80a:10043)

[15] Jude Socrates and David Whitehouse, Unramified Hilbert modular forms, with examples relating to elliptic curves. Pacific J. Math. 219 (2005), no. 2, 333-364. MR.2175121

[16] W. Stein, Explicit approaches to Abelian varieties. Ph.D. thesis at University of California at Berkeley, 2000. 
[17] Richard Taylor, On Galois representations associated to Hilbert modular forms. Invent. Math. 98 (1989), no. 2, 265-280. MR.1016264 (90m:11176)

[18] Marie France Vignéras-Guého, Le théorème d'Eichler sur le nombre de classes d'idéaux d'un corps de quaternions totalement défini et la mesure de Tamagawa. Bull. Soc. Math. France Mem., No. 37, Soc. Math. France, Paris, 1974, pp. 107-114. MR0376621 (51:12796)

Department of mathematics and Statistics, University of Calgary, 2500 University Drive N.W., Calgary, AB, Canada T2N 1N4

E-mail address: dembele@math.ucalgary.ca 